\title{
The Mechanism of Anti-Epileptogenesis by Levetiracetam Treatment is Similar to the Spontaneous Recovery of Idiopathic Generalized Epilepsy during Adolescence
}

\author{
Hiroki Kikuyama ${ }^{凶}$, Tadahito Hanaoka, Tetsufumi Kanazawa, Yasushi Yoshida, \\ Takafumi Mizuno, Hirotaka Toyoda, and Hiroshi Yoneda \\ Department of Neuropsychiatry, Osaka Medical College, Osaka, Japan
}

Objective The anti-epileptogenic drug levetiracetam has anticonvulsant and anti-epileptogenesis effects. Synergy between cell death and inflammation can lead to increased levels of apoptosis inhibitory factors and brain-derived neurotrophic factor, aberrant neurogenesis and extended axon sprouting. Once hyperexcitation of the neural network occurs, spontaneous seizures or epileptogenesis develops. This study investigated whether the anti-epileptogenic effect of levetiracetam is due to its alternate apoptotic activity.

Methods Adult male Noda epileptic rats were treated with levetiracetam or vehicle control for two weeks. mRNA quantification of Bax, Bcl-2 and GAPDH expression were performed from prefrontal cortex and hippocampus tissue samples.

Results The levetiracetam-treated group showed a significant increase of Bax/Bcl-2 mRNA expression ratio in the prefrontal cortex than the control group, but no change in the Bax/Bcl-2 mRNA expression ratio in hippocampus.

Conclusion Idiopathic generalized epilepsy including childhood absence epilepsy develop at childhood and recover spontaneously during adolescence. The aberrant neural excitable network is pruned by a neural-maturing action. This study suggests the mechanism of acquired anti-epileptogenesis by levetiracetam treatment may be similar to spontaneous recovery of idiopathic generalized epilepsy during adolescence.

Psychiatry Investig 2017;14(6):844-850

Key Words Levetiracetam, Apoptosis, Epileptogenesis, Idiopathic generalized epilepsy, Noda epileptic rat.

\section{INTRODUCTION}

Idiopathic generalized epilepsy, such as childhood absence epilepsy, develop at childhood and recover spontaneously during puberty, patients with other types of epilepsy are often prescribed medicine throughout their lifetime. Available anticonvulsant drugs may prevent seizures, but do not cure epileptogenesis. ${ }^{1,2}$ The antiepileptic action of most anticonvulsants acts on ion-channels. Many ion-channel related genes have been identified as susceptibility genes for epilepsy. ${ }^{3} \mathrm{Al}-$

Received: October 5, 2016 Revised: November 24, 2016

Accepted: December 19, 2016 Available online: August 29, 2017

$\bowtie$ Correspondence: Hiroki Kikuyama, MD

Department of Neuropsychiatry, Osaka Medical College, 2-7 Daigakumachi, Takatsuki, Osaka 569-8686, Japan

Tel: +81-726-83-1221, Fax: +81-726-83-4810

E-mail: kikuyama@osaka-med.ac.jp

(a) This is an Open Access article distributed under the terms of the Creative Commons Attribution Non-Commercial License (http://creativecommons.org/licenses/by$\mathrm{nc} / 4.0$ ) which permits unrestricted non-commercial use, distribution, and reproduction in any medium, provided the original work is properly cited. though seizures are inhibited during anticonvulsant treatment, they can occur again following cessation of treatment. Therefore, the action of ion-channels is related to the seizure, but not to epileptogenesis itself. It was previously suggested that regulation of ion-channels and neurotransmitters involved an antiictogenic action, whereas neurotransmitters, neurotrophins, apoptosis, or nitric oxide synthase may contribute to the antiepileptogenic effect. ${ }^{4}$ In temporal lobe epilepsy (TLE), a type of symptomatic localization-related epilepsy, evidence increasingly suggests that changes in the neural network are involved in acquiring epileptogenesis because of the limited focus region in the inner surface of the temporal lobe, such as the hippocampus. Decreased volume of the hippocampus due to neuronal loss is known as hippocampal sclerosis. In addition, the neurogenesis of ectopic granular cells occurs concurrently with neuronal death, and aberrant axonal sprouting (mossy fibers) are observed in the brain. ${ }^{5}$ The principal mechanism of epileptogenesis is due to the excitability of the neural network, both by ectopic neurons or axons. ${ }^{5,6}$ The Bcl-family, known 
modulators of apoptosis, and neurotrophins may be related to aberrant neurogenesis and extended axons. Indeed, apoptosis inhibitory factors such as brain-derived neurotrophic factor (BDNF), ${ }^{7} \mathrm{Bcl}-2$ and $\mathrm{Bcl}-\mathrm{XL},{ }^{8}$ were increased in the postmortem brain of patients with TLE associated with hippocampal sclerosis.

Symptomatic localization-related epilepsy can develop after the occurrence of brain infarction, head trauma, head surgery, Alzheimer's disease, or encephalitis. During brain infarction or head trauma, injured neurons secrete adenosine triphosphate (ATP). Discharged ATP subsequently activates microglia, cells which monitor the behavior of neurons, which then secrete inflammatory cytokines, such as IL-1 $\beta$, which can induce an inflammatory repair response. ${ }^{9,10}$ In Alzheimer's disease, accumulated amyloid- $\beta$ can behave as an inflammasome, ${ }^{11}$ and induce inflammation as an innate immune defense of the brain. ${ }^{12,13}$ In addition, increased inflammatory mediators such as IL-1 $\beta$ are observed in Rasmussen encephalitis. Moreover, intractable seizures can be cured by treatment with ACTH, steroids, immunoglobulins, plasmapheresis and immunosuppressants, suggesting a relationship between inflammation, seizures, and epileptogenesis. ${ }^{14}$ Animal models of epilepsy are induced by injecting kainic acid into the brain. Kainic acid induces cell death, and the subsequent release of ATP from dead cells activates microglia to produce IL- $1 \beta$ that activates astrocytes. Alpha-2 macroglobulin secreted from activated astrocytes induces the extension of neuronal axons, and repairs damaged neurons. In addition, apoptosis may promote neurogenesis. ${ }^{15}$ The EL mouse, ${ }^{16}$ a natural model of epilepsy, develops epileptogenesis with spontaneous seizures from 10 weeks of age. Apoptosis related factors, neurotrophin, ${ }^{17}$ inflammatory cytokines ${ }^{18}$ and cell cycle components ${ }^{19}$ are significantly altered at the time of epileptogenesis. In addition, Bcl-2, and BDNF levels are increased. However, cytokine levels return to the baseline, and cell cycling is normalized at 24 weeks of age in the EL mouse.

Synergy between cell death and inflammation increases levels of apoptosis inhibitory factors and BDNF, induces aberrant neurogenesis and extension of axons, and eventually leads to a hyper excitable neural network which causes epileptogenesis. Previous studies suggest that epileptic seizures promote apoptosis and increase BDNF levels. ${ }^{20}$ Thus these sensitization phenomena may promote epileptogenesis. Functional genomic analysis by microarray demonstrated significant changes in gene expression related to cell death, acute inflammatory responses, and synaptic vesicles. ${ }^{21}$ Synaptic Vesicle 2A (SV2A) is a component factor of the synaptic vesicle, and exocytosis of neurotransmitters from vesicles is decreased in SV2A deficient mice. ${ }^{22}$ It has been reported that SV2A is decreased both in postmortem brains with $\operatorname{TLE}^{23}$ and epilepsy-associated brain tumors. ${ }^{24}$

Levetiracetam is a pyrrolidone derivative, and used as an anticonvulsant, ${ }^{25}$ which does not mediate its effects through neurotransmitters (glutaminergic acid, GABA, dopamine, serotonin) or ion-channel proteins, ${ }^{26}$ but is linked to SV2A. ${ }^{27}$ Since the efficacy of levetiracetam is decreased in SV2A-deficient mice, it is likely that the clinical efficacy of this compound is related to the modulation of neurotransmitters via SV2A. ${ }^{22}$ Levetiracetam has been shown to have both anticonvulsant efficacy and antiepileptogenic action in animal models, such as the spontaneous epileptic rat (SER). ${ }^{2}$ SER was established as a double mutant by crossing tremor rats and zitter rats. SER were pretreated with levetiracetam before developing epileptogenesis to maintain anticonvulsant efficacy when stopping levetiracetam treatment, as seizures have been observed immediately after discontinuing treatment with other anticonvulsants, ${ }^{28}$ In addition, pre-treatment may decrease the number and duration of seizures, even after stopping the levetiracetam treatment. ${ }^{29}$ It has been reported that the progression of kindling was markedly delayed in levetiracetam-pretreated amygdala kindling rats due to the inhibition of increasing levels of $\mathrm{BDNF}^{30}$ It may be predicted that $\mathrm{Bcl}-2$ expression is also inhibited if levetiracetam inhibits BDNF, as the antiepileptogenic action of levetiracetam is due to the decreased expression of $\mathrm{BDNF}$ and $\mathrm{Bcl}-2$. However, as the ratio of $\mathrm{Bax} / \mathrm{Bcl}-2$ expression levels is an indispensable biomarker for the inhibition of apoptosis, the primary endpoint for the current study was the ratio of Bax/Bcl-2 mRNA expression in the prefrontal cortex and hippocampus after levetiracetam treatment of Noda epileptic rats (NER). NER is a natural model of Epilepsy, spontaneous convulsions are controlled by autosomal recessive gene for epilepsy, therefore, they are comparable to generalized epilepsy for humans. ${ }^{31}$

\section{METHODS}

\section{Animals and drug administration procedure}

Adult male Noda epileptic rats aged 5 weeks were housed under a 12 -h light-dark cycle at a temperature of $19-20^{\circ} \mathrm{C}$ with free access to both food and water. All procedures involving animals were performed in accordance with Animal Care Guidelines and approved by the Osaka Medical College Committee on Animal Care and Supply. After 1 week of acclimatization, rats were divided into two groups ( $n=6$ per group). The rats were given daily inter-peritoneal (i.p.) drug injections for two weeks. Group 1 received levetiracetam $(80 \mathrm{mg} / \mathrm{kg})$ and group 2 received a vehicle control ( $0.9 \%$ saline).

\section{RNA isolation and CDNA synthesis}

Brains were dissected and placed on an ice-cold plate. The 
samples from the prefrontal cortex and hippocampus were rapidly dissected and immediately frozen in liquid nitrogen and stored at $-80^{\circ} \mathrm{C}$. Total RNAs were isolated from the tissues using a Qiagen RNeasy Minikit (Qiagen, Hilden, Germany), and subjected to first-strand cDNA synthesis with the reverse transcriptase (RT) OMNISCRIPT (Qiagen), using a random primer (Invitrogen, Carlsbad, CA, USA) according to the manufacturer's protocol. A total RNA of 200ng (based on absorbance) was transcribed into cDNA and added to each reaction of the Quantitative RT-PCR assay.

\section{Primers and probes}

Quantification of the mRNA expression for Bax, Bcl-2 and GAPDH were performed using the LightCycler apparatus (Roche Diagnostics, Basel, Switzerland). Primers and probes used were designed with the assistance of Oligo 4.0 (National Biosciences, Plymouth, MN, USA) and synthesized by NihonIdenshiken (Sendai, Japan).

\section{Construction of the plasmid calibrators}

The transcripts that encode for Bcl-2, Bax and GAPDH were amplified by RT-PCR using specific primers. The PCR product of each gene was cloned into the pGEM T easy Vector (Promega, Madison, WI, USA) and sequenced. The standard curves were generated from serially diluted solutions of each plasmid clone as templates.

\section{Data analysis}

Statistical comparison of data from levetiracetam and saline-treated groups was performed using a two-tailed Student's t-test. A value of $\mathrm{p}<0.05$ was considered statistically significant.

\section{RESULTS}

\section{Primary endpoint}

The Levetiracetam-treated group showed a significant increase in $\mathrm{Bax} / \mathrm{Bcl}-2$ mRNA expression ratios from the prefrontal cortex compared with the control group (Mean \pm SD; $1.40 \pm$ 0.31 vs. $0.96 \pm 0.14$ respectively, $\mathrm{p}=0.02$ ) (Figure 1 ). However, levetiracetam treatment did not alter the Bax/Bcl-2 mRNA expression ratio in the hippocampus compared with the control group $(0.78 \pm 0.17$ vs. $0.80 \pm 0.27$ respectively, $p=0.90)$.

\section{Secondary endpoint}

The levetiracetam-treated group showed an increase in Bcl2 mRNA expression levels in the prefrontal cortex compared with the control group (relative values when the expression of GAPDH mRNA is regarded as 1,$000 ; 8.7 \pm 2.5$ vs. $6.2 \pm 1.8$ respectively, $\mathrm{p}=0.08$ ) (Figure 2). Levetiracetam treatment did not alter Bax mRNA expression levels compared to the control group ( $8.2 \pm 0.8$ vs. $8.1 \pm 1.0$ respectively, $\mathrm{p}=1.00$ ). In the hippocampus there was no differences in the mRNA expression level of Bax and Bcl-2 between the levetiracetam-treated and control groups (Bcl-2: 15.0 \pm 4.3 vs. $14.6 \pm 5.2$ respectively, $\mathrm{p}=0.90$; Bax: $11.2 \pm 2.0$ vs. $10.6 \pm 0.9$; respectively, $\mathrm{p}=0.48$ ).

\section{DISCUSSION}

The current study observed increased $\mathrm{Bax} / \mathrm{Bcl}-2$ ratios at the prefrontal cortex in Noda epileptic rat, which likely contribute to a decrease of $\mathrm{Bcl}-2$ rather than an increase in Bax levels. However, this association was only evaluated by analyzing mRNA expression of Bax and Bcl-2, whereas down-stream apoptosis exective factors such as caspases were not analyzed. In addition, we did not perform nuclear fragmentation analysis, a marker for apoptotic cell death, or monitor behavioral
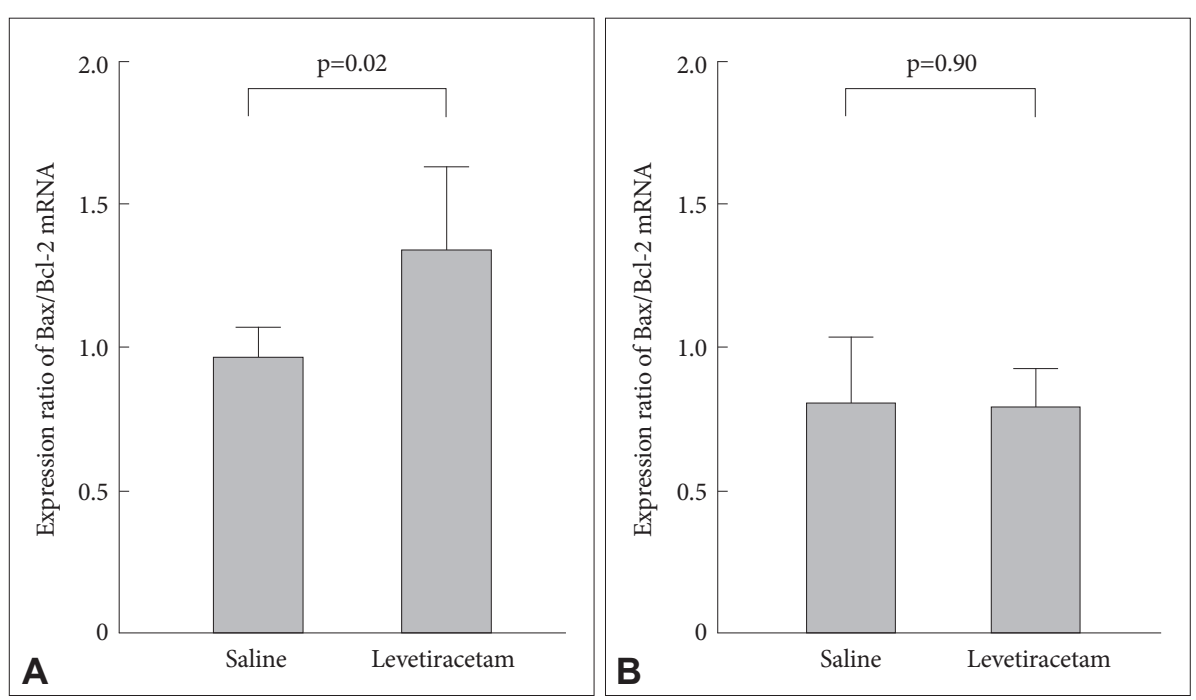

Figure 1. Expression ratio of $\mathrm{Bax} / \mathrm{Bcl}-2$ mRNA (primary endpoint). A: Prefrontal cortex. B: Hippocampus.

846 Psychiatry Investig 2017;14(6):844-850 
Figure 2. Expression level of Bax or Bcl2 mRNA (secondary endpoint). A: Bcl-2 mRNA expression level in prefrontal cortex. B: Bax mRNA expression level in prefrontal cortex. C: Bcl- 2 mRNA expression level in hippocampus. D: Bax mRNA expression level in hippocampus.
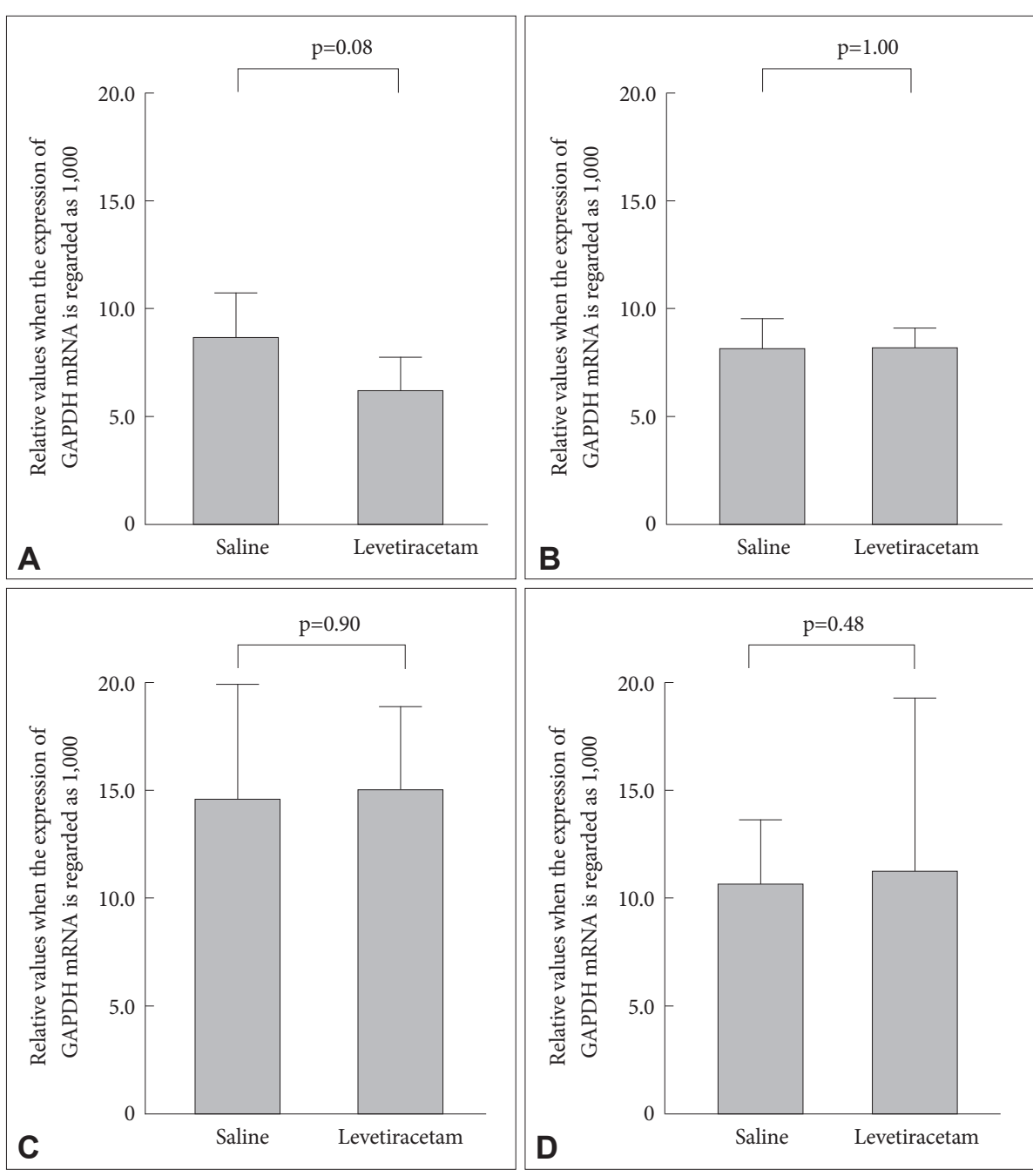

influences. Although our current findings should be regarded as preliminary, further study is required to determine the relationship between altered mRNA levels, expression of proteins, and fragmentation of the nucleus as apoptosis regulating/performance factors with the inhibition of seizures or antiepileptogenesis effect. Furthermore, the Bax/Bcl-2 ratio was up-regulated in the prefrontal cortex of levetiracetam-treated rats, whereas it was not observed in the hippocampus. Compared with the Wistar rat, SV2A expression in the parietaltemporal cortex was significantly increased in Noda epileptic rats, ${ }^{32}$ but not in the hippocampus. Levetiracetam is reported to have a diminished clinical efficacy on decreasing SV2A expression. Thus, levetiracetam may not have an effect on the apoptosis regulating factor in Noda epileptic rat hippocampus, as the SV2A expression level is same as normal rats. Although the volume and duration of levetiracetam-treatment was determined according to a previous study, ${ }^{28}$ the anti-epileptogenesis effect of levetiracetam may be different in other strains of rat, and therefore the volume and duration used in this study were not optimized for the Noda epileptic rat. The transition of $\mathrm{Bax} / \mathrm{Bcl}-2$ expression required to induce antiepileptogenesis in the EL mouse was previously reported, and the expression pattern differed between the frontal lobe cortex and hippocampus. ${ }^{17}$ Further analysis is needed to assess the expression pattern of $\mathrm{Bax}, \mathrm{Bcl}-2, \mathrm{Bcl}-\mathrm{XL}$, and other apoptosis-regulating factors throughout the duration of the treatment.

\section{The anti-epileptogenesis mechanism of levetiracetam}

Focal cortical dysplasia is a brain malformation with hyperplasia in the cortex, which induces intractable epilepsy. The mechanism of cortex hyperplasia involves the inhibition of apoptosis, increased BDNF levels, and the formation of an aberrant neuronal network. Assessment of dysplastic cells from cortical dysplasia patients demonstrated that the expression of the NR2A subunit was decreased to a quarter of the control level, whereas the NR2B subunit was increased ten times, and therefore the NR2A/NR2B ratio was $1: 40 .^{33}$

The NMDA receptor expression pattern is different accord- 
ing to the developmental stage of the brain. The main subunits are NR2A and NR2B, and the NR2A/NR2B ratio is low in infants and higher in adolescent years, whereas it becomes lower than adolescent at young adult years on human postmortem brain. ${ }^{34}$ The NR2A subunit does not have a nitric oxide synthase (NOS)-binding site, although it is present in NR2B. NOS produces nitric oxids (NO), a type of gas, and therefore it can circulate easily throughout the brain and activate guanylate cyclase (GC) present in the surrounding brain cells.

The activity of GC is 50 times higher in NR2B than NR2A. GC is an enzyme which mediates GTP to cGMP transformation. cGMP activates protein kinase $\mathrm{G}$ (PKG), by phosphorylating Ser9 in glycogen synthase kinase-3 beta (GSK-3 $\beta$ ) which inhibits activation of GSK-3 $\beta$. GSK-3 $\beta$ is involved neuroprotective effect in the human brain, and its inhibition allows increased levels of BDNF and Bcl-2, which lead to inhibition of apoptosis, and the start of neurogenesis. Hyperplasia in the brain cortex during focal cortical dysplasia may be due to the excessive neuroprotective effect afforded by the diminished NR2A/NR2B ratio. ${ }^{33}$ While the lower NR2A/NR2B ratio in infants is neuroprotective, the higher ratio in adolescents is regarded as neuropathic. The realignment of the immature brain is thought to be due to the pruning of unnecessary neural networks, to ensure the mature brain consists of only high-quality neurons. Therefore, the higher NR2A/NR2B ratio in adolescents is considered a neuron-maturing effect.
With idiopathic generalized epilepsy, such as childhood absence epilepsy, the illness spontaneously recovers during puberty. This may be due to an increased NR2A/NR2B ratio, which directs aberrant excitable neural networks to be pruned at that time. Neuroprotection or neuron-maturing is determined by the activation or suppression of GSK-3 $\beta$. GSK-3 $\beta$ is neuroprotective when suppressed, but neuron-maturing when activated. GSK-3 $\beta$ is activated following phosphorylation at Tyr 216, or suppressed by phosphorylation at Ser 9. The default status of GSK-3 $\beta$ is activated. Without stimulation, or with an inappropriate input into the neuron, GSK-3 $\beta$ remains activated by autophosphorylation, and therefore the neuron will undergo apoptosis or sub-lethal apoptosis. ${ }^{35}$ Conversely, the upstream kinase of GSK-3 $\beta$ can phosphorylate Ser9 and upon the appropriate input, the neuron will survive. The upstream cascade of GSK-3 $\beta$ contains cytokine pathways, G protein-coupled receptors (GPCR), ion channels, and the Wnt pathway (Figure 3). Various input stimuli can activate GSK-3 $\beta$ and induce cell death. However, the range between appropriate or inappropriate input stimuli is different between diseases or ages (Figure 4 ).

During infancy, the range of input stimuli is judged as appropriate for neuroprotective efficiency due to the high NR2A/ NR2B ratio. Conversely, neuron-maturing efficiency is increased during adolescence due to the changed range to judge the input stimuli as inappropriate (Figure 4A). This may sug-

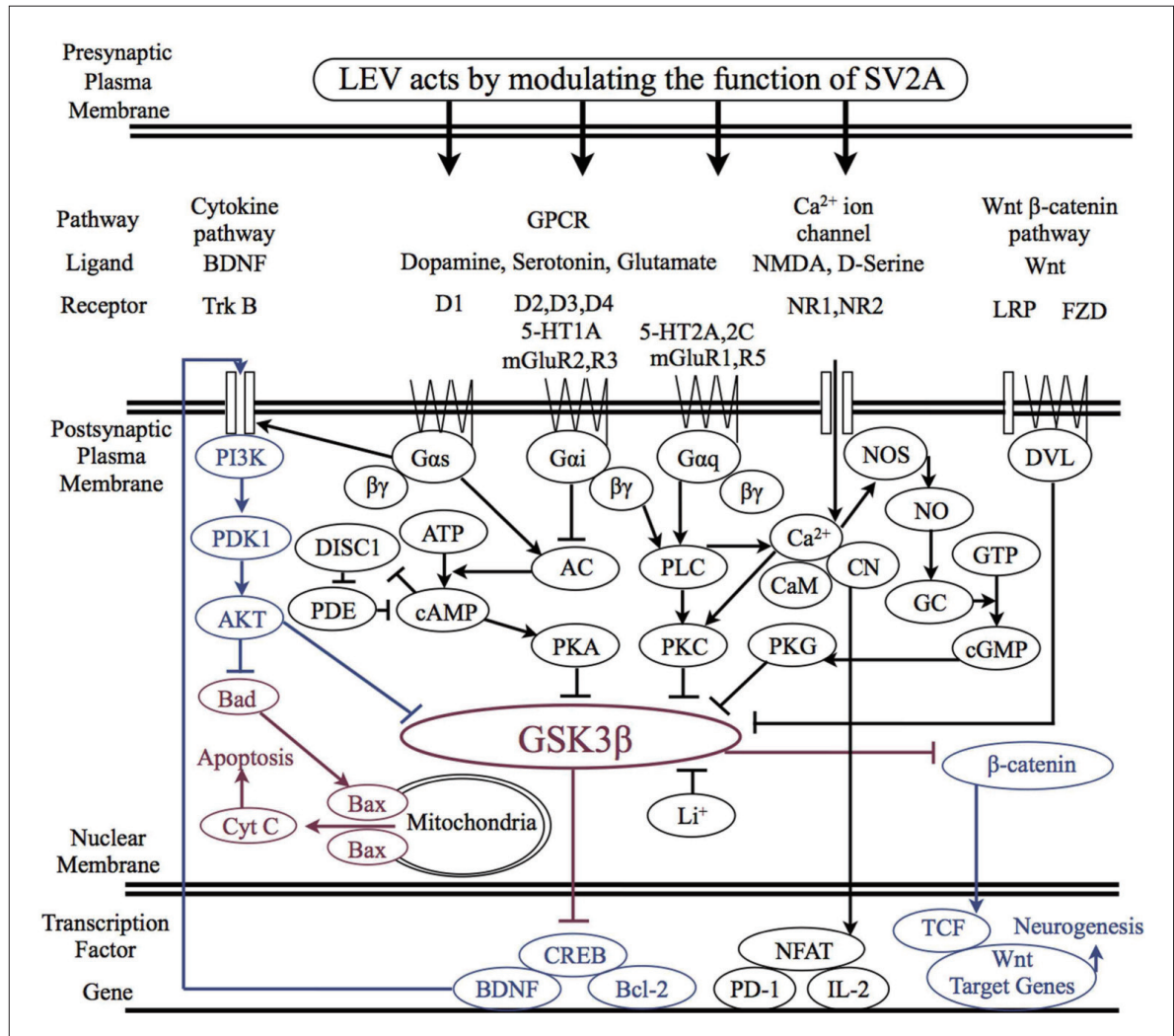

Figure 3. The intracellular signal transduction course which it is assumed to be associated with SV2A accommodation and nervous system maturity action of Levetiracetam. Arrow: promotion, T shape bar: inhibition, Red color: activated, Blue color: hypofunction. AC: adenylyl cyclase, ATP: adenosine triphosphate, BDNF: brain-derived neurotrophic factor, cAMP: cyclic adenosine monophosphate, CaM: calmodulin, cGMP: cyclic guanosine monophosphate, $\mathrm{CN}$ : calcineurin, CREB: CAMP response element binding protein, Cyt C: cytochrome C, DISC1: disrupted schizophrenia 1, DVL: mammalian homologue of drosophila dishevelled, EGF: epidermal growth factor, GC: guanylyl cyclase, GTP: guanosine triphosphate, FZD: frizzled, GSK3 $\beta$ : glycogen synthase kinase $3 \beta$, GPCR: G protein-coupled receptor, IL-2: interleukin 2, LRP: LDL- receptor-related protein, NFAT: nuclear factor of activated T cells, NO: nitric oxidase, NOS: nitric oxidase synthase, PDE: phospho-di-esterase, PDK1: phosphatidylinositide-dependent kinase 1, PD-1: programmed deth 1, PI3K: phosphatidylinositol 3 kinase, PKA: protein kinase $A, P K C$ : protein kinase C, PKG: protein kinase G, PLC: phospholipase C, TCF: T-cell factor. 


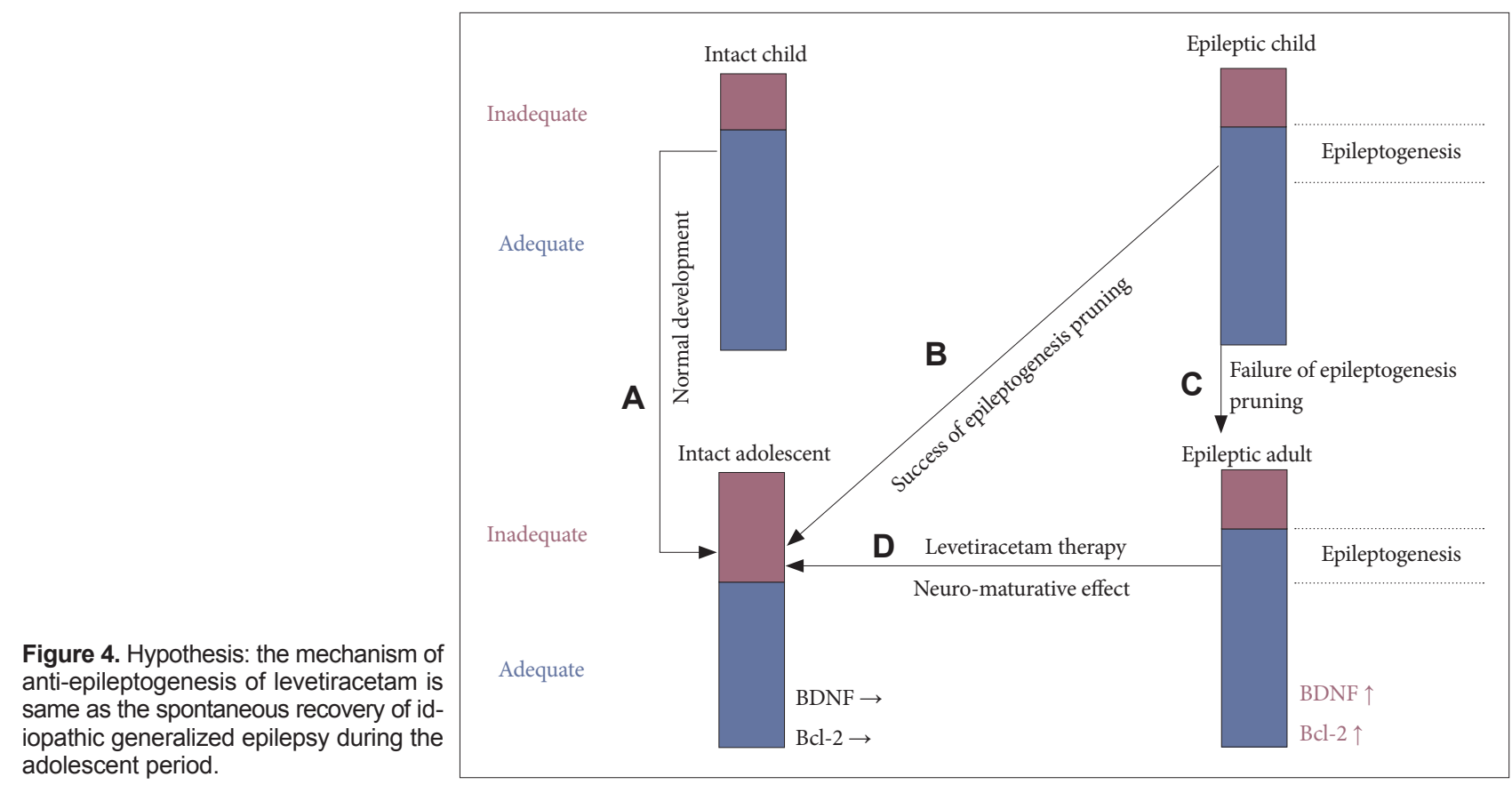

gest why idiopathic generalized epilepsy spontaneously resolves during the realignment of the adolescent brain network. Simply, the aberrant neural excitable network is pruned by a neural-maturing action (Figure 4B). When spontaneous recovery does not occur, it is likely that the neuron-maturing action has not been activated (Figure 4C). The NR2A/NR2B ratio decreases again after adolescence and therefore idiopathic generalized epilepsy is not easily cured in adults if the aberrant neural excitable network is not pruned at adolescence.

Further discussion is warranted for the relationship between neurotransmitters and neuro-developmental action. It is thought that levetiracetam may change the volume of discharge of neurotransmitters from the pre-synaptic cell to the synaptic cleft through its action on SV2A. Neurotransmitters, such as dopamine and noradrenalin are known to be neuroprotective if the quantity of antipsychotic agents is a minimal, ${ }^{36}$ although neurodeficiency will occur if it is excessive. ${ }^{37}$ In epileptic patients, higher BDNF and Bcl-2 levels provide a neuroprotective effect similar to that seen in childhood, and therefore gives a wider range to evaluate the input stimuli as appropriate. Levels of neurotransmitters released from the pre-synaptic neuron are changed by levetiracetam-treatment and cause post-synaptic neurons to undergo neuro-maturation. This pruning of the aberrant excitable network exerts the anti-epileptogenesis effect of levetiracetam treatment (Figure 4D). We therefore suggest that the mechanism of acquired anti-epileptogenesis by levetiracetam treatment is the same as the spontaneous recovery seen in idiopathic generalized epilepsy during adolescence. The current findings verify our assertions, although the process of GSK- $3 \beta$ activation following the volumetric alterations of neu- rotransmitter release within the synaptic cleft is not well established. Therefore further research is needed to determine which neurotransmitter and which receptor are involved in the process (Figure 3).

\section{Acknowledgments}

We are grateful to Dr. T. Nakagawa for his helpful advice and to UCB S. A. levetiracetam supply.

\section{REFERENCES}

1. Loscher W. Current status and future directions in the pharmacotherapy of epilepsy. Trends Pharmacol Sci 2002;23:113-118.

2. Sasa M. A new frontier in epilepsy: novel antiepileptogenic drugs. J Pharmacol Sci 2006;100:487-494.

3. Armijo JA, Shushtarian M, Valdizan EM, Cuadrado A, de las Cuevas I, Adin J. Ion channels and epilepsy. Curr Pharm Des 2005;11:1975-2003.

4. Weaver DF. Epileptogenesis, ictogenesis and the design of future antiepileptic drugs. Can J Neurol Sci 2003;30:4-7.

5. Koyama R, Yamada MK, Fujisawa S, Katoh-Semba R, Matsuki N, Ikegaya Y. Brain-derived neurotrophic factor induces hyperexcitable reentrant circuits in the dentate gyrus. J Neurosci 2004;24:7215-7224.

6. Scharfman H, Goodman J, McCloskey D. Ectopic granule cells of the rat dentate gyrus. Dev Neurosci 2007;29:14-27.

7. Wang FJ, Li CM, Hou XH, Wang XR, Zhang LM. Selective Upregulation of Brain-derived Neurotrophic Factor (BDNF) Transcripts and BDNF Direct Induction of Activity Independent N-Methyl-D-aspartate Currents in Temporal Lobe Epilepsy Patients with Hippocampal Sclerosis. J Int Med Res 2011;39:1358-1368.

8. Engel T, Henshall DC. Apoptosis, Bcl-2 family proteins and caspases: the ABCs of seizure-damage and epileptogenesis? Int J Physiol Pathophysiol Pharmacol 2009;1:97-115.

9. John GR, Lee SC, Song X, Rivieccio M, Brosnan CF. IL-1-regulated responses in astrocytes: relevance to injury and recovery. Glia 2005;49: 161-176.

10. Kono H, Rock KL. How dying cells alert the immune system to danger. Nat Rev Immunol 2008;8:279-289. 
11. Schroder K, Tschopp J. The inflammasomes. Cell 2010;140:821-832.

12. Eisenstein M. Genetics: finding risk factors. Nature 2011;475:S20-S22.

13. Rock KL, Latz E, Ontiveros F, Kono H. The sterile inflammatory response. Annu Rev Immunol 2010;28:321-342.

14. Vezzani A, Balosso S, Maroso M, Zardoni D, Noe F, Ravizza T. ICE/caspase 1 inhibitors and IL-1beta receptor antagonists as potential therapeutics in epilepsy. Curr Opin Investig Drugs 2010;11:43-50.

15. Ryoo HD, Gorenc T, Steller H. Apoptotic cells can induce compensatory cell proliferation through the JNK and the Wingless signaling pathways. Dev Cell 2004;7:491-501.

16. Meidenbauer JJ, Mantis JG, Seyfried TN. The EL mouse: a natural model of autism and epilepsy. Epilepsia 2011;52:347-357.

17. Murashima YL, Suzuki J, Yoshii M. Developmental program of epileptogenesis in the brain of EL mice. Epilepsia 2005;46(Suppl 5):10-16.

18. Murashima YL, Suzuki J, Yoshii M. Role of cytokines during epileptogenesis and in the transition from the interictal to the ictal state in the epileptic mutant EL mouse. Gene Regul Syst Bio 2008;2:267-274.

19. Murashima YL, Suzuki J, Yoshii M. Cell cycle reentry and cell proliferation as candidates for the seizure predispositions in the hippocampus of EL mouse brain. Epilepsia 2007;48(Suppl 5):119-125.

20. Bozzi Y, Dunleavy M, Henshall DC. Cell signaling underlying epileptic behavior. Front Behav Neurosci 2011;5:45.

21. Muller FJ, Laurent LC, Kostka D, Ulitsky I, Williams R, Lu C, et al. Regulatory networks define phenotypic classes of human stem cell lines. Nature 2008;455:401-405.

22. Kaminski RM, Gillard M, Leclercq K, Hanon E, Lorent G, Dassesse D, et al. Proepileptic phenotype of SV2A-deficient mice is associated with reduced anticonvulsant efficacy of levetiracetam. Epilepsia 2009;50: 1729-1740.

23. van Vliet EA, Aronica E, Redeker S, Boer K, Gorter JA. Decreased expression of synaptic vesicle protein $2 \mathrm{~A}$, the binding site for levetiracetam, during epileptogenesis and chronic epilepsy. Epilepsia 2009;50: 422-433.

24. de Groot M, Toering ST, Boer K, Spliet WG, Heimans JJ, Aronica E, et al. Expression of synaptic vesicle protein $2 \mathrm{~A}$ in epilepsy-associated brain tumors and in the peritumoral cortex. Neuro Oncol 2010;12:265-273.

25. Schulze-Bonhage A. Brivaracetam for the treatment of epilepsy. Expert Opin Pharmacother 2011;12:1959-1966.

26. Noyer M, Gillard M, Matagne A, Henichart JP, Wulfert E. The novel antiepileptic drug levetiracetam (ucb L059) appears to act via a specific binding site in CNS membranes. Eur J Pharmacol 1995;286:137-146.

27. Lynch BA, Lambeng N, Nocka K, Kensel-Hammes P, Bajjalieh SM,
Matagne A, et al. The synaptic vesicle protein SV2A is the binding site for the antiepileptic drug levetiracetam. Proc Natl Acad Sci U S A 2004; 101:9861-9866.

28. Ji-qun C, Ishihara K, Nagayama T, Serikawa T, Sasa M. Long-lasting antiepileptic effects of levetiracetam against epileptic seizures in the spontaneously epileptic rat (SER): differentiation of levetiracetam from conventional antiepileptic drugs. Epilepsia 2005;46:1362-1370.

29. Yan HD, Ji-qun C, Ishihara K, Nagayama T, Serikawa T, Sasa M. Separation of antiepileptogenic and antiseizure effects of levetiracetam in the spontaneously epileptic rat (SER). Epilepsia 2005;46:1170-1177.

30. Husum H, Bolwig TG, Sanchez C, Mathe AA, Hansen SL. Levetiracetam prevents changes in levels of brain-derived neurotrophic factor and neuropeptide Y mRNA and of Y1- and Y5-like receptors in the hippocampus of rats undergoing amygdala kindling: implications for antiepileptogenic and mood-stabilizing properties. Epilepsy Behav 2004;5: 204-215.

31. Noda A, Hashizume R, Maihara T, Tomizawa Y, Ito Y, Inoue M, et al. NER rat strain: a new type of genetic model in epilepsy research. Epilepsia 1998;39:99-107.

32. Okumura H, Terada R, Ishihara S, Kitataku R, Tomita T, Tokudome K, et al. Expression analysis of SV2A and SNARE-related proteins in Noda epileptic rat (NER), a rat model of generalized tonic-clonic seizure. Joint congress of the 21st congress of Japanese society of clinical neuropsychopharmacology and the 41st annual meeting of Japanese society of neuropsychopharmacology. Tokyo, Japan, 2011, p.178.

33. Crino PB, Duhaime AC, Baltuch G, White R. Differential expression of glutamate and GABA-A receptor subunit mRNA in cortical dysplasia. Neurology 2001;56:906-913.

34. Law AJ, Weickert CS, Webster MJ, Herman MM, Kleinman JE, Harrison PJ. Expression of NMDA receptor NR1, NR2A and NR2B subunit mRNAs during development of the human hippocampal formation. Eur J Neurosci 2003;18:1197-1205.

35. Jope RS, Roh MS. Glycogen synthase kinase-3 (GSK3) in psychiatric diseases and therapeutic interventions. Curr Drug Targets 2006;7:14211434.

36. Noh JS, Kim EY, Kang JS, Kim HR, Oh YJ, Gwag BJ. Neurotoxic and neuroprotective actions of catecholamines in cortical neurons. Exp Neurol 1999;159:217-224.

37. Qing H, Xu H, Wei Z, Gibson K, Li XM. The ability of atypical antipsychotic drugs vs. haloperidol to protect PC12 cells against MPP+-induced apoptosis. Eur J Neurosci 2003;17:1563-1570. 